\title{
Acute kidney injury in patients with Kawasaki disease
}

\author{
Gwo-Tsann Chuang', I-Jung Tsai', Ming-Tai Lin' and Luan-Yin Chang'
}

BACKGROUND: Kawasaki disease was well known for coronary artery abnormalities with few reports of incidence of acute kidney injury (AKI). Our aim was to identify the rate of AKI in patients with Kawasaki disease and its associated factors. METHODS: All patients with Kawasaki disease admitted to a medical center from February 2004 to August 2014 were evaluated. Data collection included serum creatinine level, serial echocardiography reports, white blood cell count, C-reactive protein level, alanine transaminase level, urine white blood cell count, and renal ultrasound reports if available. AKI was defined when a patient's serum creatinine level was higher than 1.5 times upper limits of age-specific serum creatinine levels.

RESULTS: This cohort study included 332 patients (191 boys and 141 girls; aged 0.12 to 11.3 y, median 1.39 y) and 93 patients (28\%) of them had AKI. Multivariate logistic regression revealed that age and alanine transaminase level were significantly associated with AKI (odds ratio (OR): 0.521, 95\% confidence interval (CI): $0.377-0.718, P<0.001$, and OR: 1.003, 95\% Cl: $1.000-1.005, P=0.017$, respectively).

CONCLUSION: This study demonstrated that AKI exists in substantial proportion of patients with KD. Young age and high alanine transaminase level are the main associated factors for AKI in these patients.

K awasaki disease (KD), also known as mucocutaneous lymph node syndrome, is predominantly a disease occurring in childhood which consists of medium-vessel vasculitis $(1,2)$. The most important concern is coronary artery aneurysm formation, which may be fatal if left untreated. However, there were only few reports on renal injury in patients with $\mathrm{KD}$.

With nuclear imaging technology, renal involvement was reported in $52 \%$ of the KD patients, but acute kidney injury (AKI) defined by serum creatinine elevation was very uncommon (3). Only few cases of KD patients with AKI underwent renal biopsy, and pathology revealed tubulointerstitial nephritis (4-6) or hemolytic uremic syndrome (7). However, pediatric patients with renal injury may be underestimated due to absence of clear definition and lack of age-specific normal values of serum creatinine. This is especially true among patients with KD, since the peak incidence falls below $2 \mathrm{y}$ old. In response to the advent of recently developed age-specific reference for serum creatinine level in pediatric population (8), this study aims to identify the rate and factors associated with renal injury in $\mathrm{KD}$ patients.

\section{RESULTS}

From February 2004 to August 2014, a total of 356 patients were enrolled consecutively in this study. Among them, 13 patients did not receive checks on serum creatinine level upon admission, 2 patients lacked cardiac echo exam reports, 6 patients had coincident urinary tract infection, one patient had congenital small kidneys, and 2 patients suffered from Kawasaki disease shock syndrome. They were excluded from subsequent analyses.

The remaining 332 patients (191 boys and 141 girls; aged 0.12 to $11.3 \mathrm{y}$, median $1.39 \mathrm{y}$ ) were further analyzed (Table 1 ). Infants below 6 mo old represented $16.0 \%$ of the cases, whereas the majority (52.4\%) of the patients were between 6 mo old to 2 y old. Ninety-three patients (28.0\%) had AKI among which 7 patients (2.1\%) had more severe renal injury, with serum creatinine level higher than two times of normal upper limit. The age distribution of KD patients with or without AKI was shown in Figure 1. For patients under $1 \mathrm{y}$ old, the incidence of AKI was $47.1 \%$, which was much higher than those over $1 \mathrm{y}$ old $(17.4 \% ; P<0.01)$.

Among the 331 patients who received checks on C-reactive protein, the level was elevated (median $6.00,0.01-26.50 \mathrm{mg} /$ dl) and $80.7 \%$ of the patients levelled above $3 \mathrm{mg} / \mathrm{dl}$. Among the 266 patients who received checks on alanine transaminase, the median level was 37 (3-909) U/l, with $48.1 \%$ of the patients levelling above $40 \mathrm{U} / \mathrm{l}$. Among the 279 patients with urinalysis data, $31.5 \%$ had sterile pyuria, which was defined as urine white blood cell count equal or more than 10/HPF. Among 59 patients who received renal ultrasound, $57.6 \%$ had nephromegaly.

All patients received treatments with intravenous immunoglobulin and antiplatelet medication. By performing serial echocardiography, $21.7 \%$ had coronary artery abnormalities, including $6.3 \%$ coronary artery aneurysm and $15.4 \%$ coronary ectasia. For patients with coronary artery aneurysm, antiplatelet medications were kept for longer periods as needed.

In univariate analysis, age (median 0.88 , range $0.12-4.67$ ) in AKI group was significantly $(P<0.01)$ lower than that (median

'Department of Pediatrics, National Taiwan University Hospital, College of Medicine, National Taiwan University, Taipei, Taiwan. Correspondence: Luan-Yin Chang (lychang@ntu.edu.tw) 
Table 1. Clinical features, treatment, and outcome of 332 patients with KD

\begin{tabular}{|c|c|}
\hline Variables & $n(\%)$ or median (range) \\
\hline Male/female & $191 / 141(57.5 \% / 42.5 \%)$ \\
\hline Age (years) & $1.39(0.12-11.3)$ \\
\hline \multicolumn{2}{|l|}{ Age category } \\
\hline$<0.5 y$ & $53(16.0 \%)$ \\
\hline $0.5-2 y$ & $174(52.4 \%)$ \\
\hline$>2 y$ & $105(31.6 \%)$ \\
\hline Symptoms onset to KD diagnosis (days) & $4(0-18)$ \\
\hline \multicolumn{2}{|l|}{ Laboratory data } \\
\hline 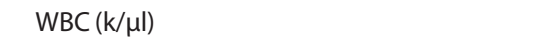 & $13.70(4.01-36.64)$ \\
\hline$W B C>15 \mathrm{k} / \mu \mathrm{l}$ & $133(40.1 \%)$ \\
\hline 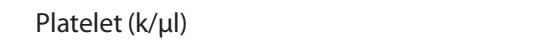 & $336(71-969)$ \\
\hline Platelet $>450 \mathrm{k} / \mu \mathrm{l}$ & $58(17.5 \%)$ \\
\hline $\mathrm{CRP}(\mathrm{mg} / \mathrm{dl})$ & $6.00(0.01-26.50)^{\mathrm{a}}$ \\
\hline $\mathrm{CRP}>3 \mathrm{mg} / \mathrm{dl}$ & $267(80.7 \%)^{\mathrm{a}}$ \\
\hline $\operatorname{ALT}(U / I)$ & $37(3-909)^{b}$ \\
\hline $\mathrm{ALT}>40 \mathrm{U} / \mathrm{l}$ & $168(48.1 \%)^{b}$ \\
\hline Urine $\mathrm{WBC} \geq 10 / \mathrm{HPF}$ & $88(31.5 \%)^{c}$ \\
\hline Nephromegaly by renal sonography & $57.6(\%)^{d}$ \\
\hline \multicolumn{2}{|l|}{ Treatment } \\
\hline $\begin{array}{l}\text { Intravenous immunoglobulin + antiplatelet } \\
\text { therapy }\end{array}$ & $332(100.0)$ \\
\hline \multicolumn{2}{|l|}{ Outcome } \\
\hline AKI & $93(28.0 \%)$ \\
\hline Coronary artery abnormality & $72(21.7 \%)$ \\
\hline Coronary artery aneurysm & $21(6.3 \%)$ \\
\hline Coronary artery ectasia & $51(15.4 \%)$ \\
\hline
\end{tabular}

Data are presented as case number, percentages, or median (range).

aBased on 331 patients with available C-reactive protein results. "'Based on 266 patients with available alanine transaminase results. 'Based on 279 patients with available urinalysis results. ${ }^{\circledR B}$ Based on 59 patients with available renal sonography results. ALT, alanine transaminase; AKI, acute kidney injury; CRP, C-reactive protein; dl, deciliter; HPF, high-power field; KD, Kawasaki disease; $\mu$ I micro-liter; WBC, white blood cell.

1.63, range $0.12-11.25$ ) in non-AKI group (Table 2); alanine transaminase level was significantly higher in AKI group than in non-AKI group $(P<0.01)$; pyuria was more prevalent in AKI group than in non-AKI group $(P<0.01)$. Other clinical or laboratory parameters did not show significant difference between the two groups.

We then performed multivariate logistic regression for factors associated with AKI revealed in univariate analysis (Table 3), and found only age and alanine transaminase level were significantly associated with AKI (odds ratio (OR): 0.521, 95\% confidence interval (CI): $0.377-0.718, P<0.001$, and OR: 1.003, 95\% CI: 1.000-1.005, $P=0.017$, respectively).

\section{DISCUSSION}

Renal involvement in patients with $\mathrm{KD}$ had been reported before (3-7); however, no study had been designed with the specific aim to explore the incidence of AKI. The present study documents that a high incidence of AKI (28.0\%) was present in patients with $\mathrm{KD}$. As KD is a disease of medium-vessel vasculitis, renal or intrarenal arterial inflammation might be the major cause of AKI. Earlier review summarized and indicated

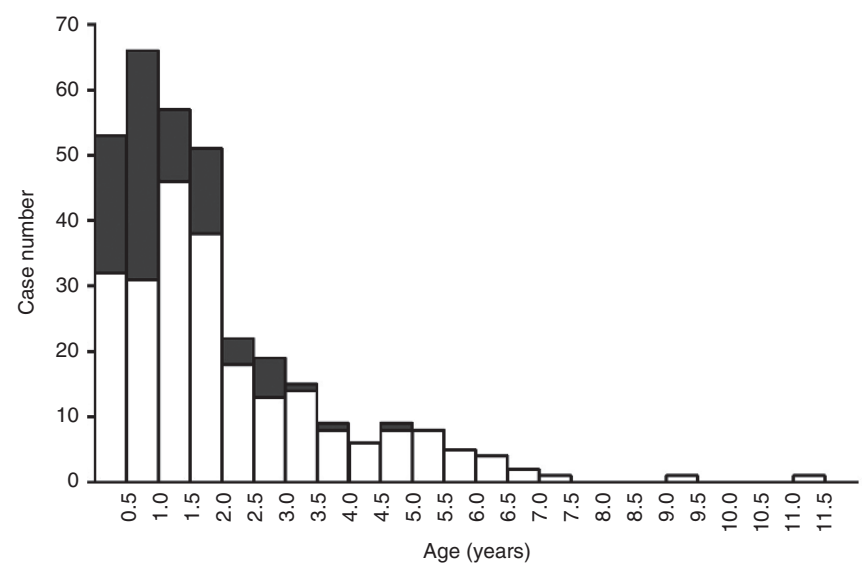

Figure 1. Age distribution of Kawasaki disease (KD) patients. The peak incidence of patients with KD is below 2 y old. Non-acute kidney injury (AKI) cases are shown in white bar and AKI cases in black bar. The proportion of KD patients with AKI is significantly higher among younger age groups, especially among infants.

that vasculitis of arteries in the kidney, immune-complexmediated renal injuries, and T-cell immune-regulatory abnormalities might be underlying mechanisms, although the precise mechanism of kidney involvement and renal injury in KD patients remains unclear (9).

There has always been difficulty in assessing renal function in pediatric population. Except in neonatal period, serum creatinine level increases gradually with age. References in the era of conventional Jaffe method should not be used nowadays, as serum creatinine level would have great difference (falsely higher in conventional Jaffe method than compensated Jaffe method for more than $0.3 \mathrm{mg} / \mathrm{dl}$ ) (10). Moreover, many serum creatinine references were inappropriate for our study group due to lack of narrow age range grouping (e.g., broader grouping such as $0-4,4-7$, and 7-10 y old). Since the peak incidence of patients with KD falls below 2 y old, therefore, we employed the results reported recently by Soeby et al. (8) to define AKI in this younger age population.

Chang et al. (11) revealed that patients with $\mathrm{KD}$ younger than 6 mo of age were more likely to have atypical presentation, and in this age group, incidence of coronary artery abnormalities was significantly higher, thus earlier recognition of KD among febrile infants is crucial. Our study demonstrated that age is significantly associated $(P<0.001$; OR: $0.521 ; 95 \%$ CI: 0.377-0.718) with AKI, which indicates a higher incidence of AKI in younger patients. Among infants with KD, the incidence of AKI is $47.1 \%$. Therefore, we suggest measurement of serum creatinine level in infants and children with KD for early detection and appropriate management of AKI.

Huang et al. (12) reported a high incidence (70\% of 20 cases) of nephromegaly in patients with $\mathrm{KD}$ who had ever received renal ultrasound exam. A higher plasma hepatocyte growth factor (HGF) to transforming growth factor $\beta 1$ (TGF- $\beta 1$ ) ratio (HGF/TGF- $\beta 1$ ) was noted in patients with KD. Previously, a higher HGF/TGF- $\beta 1$ ratio in patients with biliary atresia was demonstrated to be associated with increased kidney volume and a higher glomerular filtration rate (13). Should the higher 
Articles | Chuangetal.

Table 2. Comparison of variables between non-AKI group and AKI group

\begin{tabular}{|c|c|c|c|}
\hline Variables & Non-AKI group $(n=239)$ & AKI group $(n=93)$ & $P$ value \\
\hline Male & $138(57.7 \%)$ & $53(57.0 \%)$ & $0.90^{\mathrm{a}}$ \\
\hline Age (years) & $1.63(0.12-11.25)$ & $0.88(0.12-4.67)$ & $<0.01^{b}$ \\
\hline 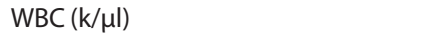 & $13.44(4.40-36.64)$ & $14.13(4.01-28.29)$ & $0.47^{b}$ \\
\hline Platelet $(\mathrm{k} / \mu \mathrm{l})$ & $336(71-969)$ & $336(123-822)$ & $0.88^{b}$ \\
\hline Urine WBC $\geq 10 / \mathrm{HPF}$ & $52(26.3 \%)(n=198)$ & $36(44.4 \%)(n=81)$ & $<0.01^{\mathrm{b}}$ \\
\hline Nephromegaly by renal sonography & $20(54.1 \%)(n=37)$ & $14(63.6 \%)(n=22)$ & $0.47^{\mathrm{a}}$ \\
\hline Coronary artery abnormality & $52(21.8 \%)$ & $20(21.5 \%)$ & $0.96^{\mathrm{a}}$ \\
\hline Coronary artery ectasia & $36(15.1 \%)$ & $15(16.1 \%)$ & $0.81^{\mathrm{a}}$ \\
\hline
\end{tabular}

Data are presented as case number, percentages, or median (range).

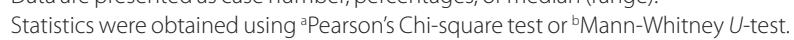

ALT, alanine transaminase; AKI, acute kidney injury; CRP, C-reactive protein; dl, deciliter; HPF, high-power field; $\mu$ l micro-liter; WBC, white blood cell.

Table 3. Multivariate logistic regression for factors associated with acute kidney injury

\begin{tabular}{lccc}
\hline Variables & OR & $95 \% \mathrm{Cl}$ & Pvalue $^{\mathrm{a}}$ \\
\hline Age (years) & 0.521 & $0.377-0.718$ & $<0.01$ \\
Alanine transaminase (U/I) & 1.003 & $1.000-1.005$ & 0.02 \\
Urine white blood cell count $\geq 10 / \mathrm{HPF}(\%)$ & 0.844 & $0.337-2.109$ & 0.72 \\
\hline aStastistics were obtained using multivariate logsitic regression. & \\
Cl, confidence interval; HPF, high-power field; OR, odds ratio. &
\end{tabular}

$\mathrm{HGF} / \mathrm{TGF}-\beta 1$ ratio be observed in the patients with $\mathrm{KD}$, nephromegaly should represent a proliferative status with a higher glomerular filtration rate and thus lower chance of AKI. However, in our current study, the incidence of nephromegaly was slightly higher in AKI group than in non-AKI group (63.6 vs. $54.6 \%, P>0.05)$. Thus, the association between nephromegaly and renal function in patients with $\mathrm{KD}$ is not clear and remains to be further explored.

Hepatic dysfunction had been documented in patients with KD (14). Eladawy et al. (15) reported $37.2 \%$ of abnormal alanine transaminase or aspartate transaminase among patients with $\mathrm{KD}$. Our study showed $48.1 \%$ of patients with KD had elevated alanine transaminase level. When comparing AKI group with nonAKI group, a slightly higher level was noted in the former group, supporting the fact that KD is a multi-organ disease, and inflammation severity of liver and kidney might be closely related.

There are some limitations of our study. First, it is a retrospective study and the baseline serum creatinine level was not checked. Therefore, we might underestimate the incidence of AKI as some patients with AKI might not be identified due to lack of the baseline level. Second, no biopsy was done to demonstrate the underlying mechanism of renal injury. Third, fractional excretion of sodium, urinary $\beta 2$-microglobulin, or gallium or $99 \mathrm{mTc}$-DMSA renal scintigraphy were not examined in this study to identify causes of AKI. However, from our present study, we strongly recommend patients with $\mathrm{KD}$ to check serum creatinine and alanine transaminase levels routinely during both acute phase and convalescent phase to evaluate if renal and hepatic injury is present.

In conclusion, this is the first study to use age-specific serum creatinine reference to evaluate renal function in pediatric patients with $\mathrm{KD}$. Contrary to previous understanding, substantial proportion of patients with KD actually has AKI, suggesting a multi-organ involvement of this disease. The associated factors of patients with KD developing AKI are young age and hepatic dysfunction.

\section{METHODS \\ Study Population}

We retrospectively reviewed children admitted to a medical center in Taipei, the National Taiwan University Hospital, who were diagnosed with acute febrile KD from February 2004 to August 2014. The exclusion criteria were: (i) lack of serum creatinine data or echocardiography reports, (ii) coincident urinary tract infection or congenital renal anomalies, or (iii) having other conditions that can directly cause acute kidney injury such as shock. The study protocol was approved by the institutional review board of National Taiwan University Hospital (201511063RIND). As a retrospective study, subject and/or parental informed consent was not obtained.

KD was defined according to AHA 2004 diagnostic criteria (16). Diagnosis of complete $\mathrm{KD}$ was based on the presence of $5 \mathrm{~d}$ of fever and four of the following five principal clinical features: (i) changes in extremities, (ii) polymorphous exanthema, (iii) bilateral bulbar conjunctival injection without exudates, (iv) changes in lips and oral cavity, and (v) cervical lymphadenopathy (diameter $>1.5 \mathrm{~cm}$ ). Patients with fever for $5 \mathrm{~d}$ but having less than four principal features were then diagnosed as having atypical KD if coronary artery disease was detected by either 2D echocardiography or coronary angiography.

Data collected include serum creatinine level, serial echocardiography reports, white blood cell count, C-reactive protein level, alanine transaminase level, urine white blood cell count, and renal ultrasound reports if available. The serum creatinine level was measured using a chemistry analyzer (Toshiba 200FR, Toshiba Medical Systems, Tokyo, Japan during 2004-2010; AU5800, Beckman Coulter, Brea, California since 2011) with compensated Jaffe method.

According to 2011 Kidney Disease: Improving Global Outcomes Clinical Practice Guideline for Acute Kidney Injury (17), AKI is defined 
as serum creatinine level elevation to more than 1.5 times of baseline level. Since our patients did not have the baseline level before diagnosis of $\mathrm{KD}$, therefore, we defined $\mathrm{AKI}$ in this study when a patient's serum creatinine level was higher than 1.5 times upper limits of normal agespecific serum creatinine reference levels as reported (8).

Echocardiography examination using a broadband transducer (ACUSON Sequoia C512, Siemens, Mountain View, CA: 8V3c transducer $(3-8 \mathrm{MHz})$ or $10 \mathrm{~V} 4 \mathrm{c}$ transducer $(4-10 \mathrm{MHz})$; or iE33, Philips, Willoughby, OH: S8-3 transducer $(3-8 \mathrm{MHz})$ or $\mathrm{S} 12-4$ transducer (4-12 MHz)) to evaluate coronary artery abnormalities was done by trained pediatric cardiologists. The results of cardiac ultrasound were classified into: (i) normal coronary arteries, (ii) coronary artery ectasia, defined by coronary artery diameter $\mathrm{Z}$ score $>+2.5$, or (iii) coronary artery aneurysm, defined by coronary artery diameter is more than 1.5 times larger than adjacent vessel segment (18).

Renal ultrasound examination using a broadband transducer (LOGIQ S6, GE Healthcare, Wauwatosa, WI: 4C transducer (1.5-4.5 $\mathrm{MHz})$ ) was done by trained pediatric nephrologists. Nephromegaly was defined by increased renal length or anteroposterior diameter to more than 2 SDs above the mean value (19).

\section{Statistical Analysis}

Shapiro-Wilk test was employed to test data distribution of all continuous variables. As all data deviated from normal distribution, thus they were presented as medians with ranges. The differences between groups were then analyzed by using Mann-Whitney $U$-test. Pearson's Chi-square test with Yates' continuity correction was used to compare frequencies. Statistical analyses were performed with SPSS for Windows, version 16.0 (released in 2007, SPSS, Chicago, IL). A probability value of $P<0.05$ was considered statistically significant.

\section{STATEMENT OF FINANCIAL SUPPORT}

No financial assistance was received and there were neither financial ties to products nor potential/perceived conflicts of interest in the study.

\section{REFERENCES}

1. Kawasaki T, Kosaki F, Okawa S, Shigematsu I, Yanagawa H. A new infantile acute febrile mucocutaneous lymph node syndrome (MLNS) prevailing in Japan. Pediatrics 1974;54:271-6.

2. Kawasaki T. [Acute febrile mucocutaneous syndrome with lymphoid involvement with specific desquamation of the fingers and toes in children]. Arerugi 1967;16:178-222.

3. Wang JN, Chiou YY, Chiu NT, Chen MJ, Lee BF, Wu JM. Renal scarring sequelae in childhood Kawasaki disease. Pediatr Nephrol 2007;22:684-9.

4. Veiga PA, Pieroni D, Baier W, Feld LG. Association of Kawasaki disease and interstitial nephritis. Pediatr Nephrol 1992;6:421-3.

5. Bonany PJ, Bilkis MD, Gallo G, et al. Acute renal failure in typical Kawasaki disease. Pediatr Nephrol 2002;17:329-31.
6. Papadodima SA, Sakelliadis EI, Goutas ND, Vlachodimitropoulos DG, Spiliopoulou CA. Atypical kawasaki disease presenting with symptoms from the genitourinary system: an autopsy report. J Trop Pediatr 2009;55:55-7.

7. Ferriero DM, Wolfsdorf JI. Hemolytic uremic syndrome associated with Kawasaki disease. Pediatrics 1981;68:405-6.

8. Søeby K, Jensen PB, Werge T, Sørensen S. Mining of hospital laboratory information systems: a model study defining age- and gender-specific reference intervals and trajectories for plasma creatinine in a pediatric population. Clin Chem Lab Med 2015;53:1621-30.

9. Watanabe T. Kidney and urinary tract involvement in kawasaki disease. Int J Pediatr 2013;2013:831834.

10. Ghasemi A, Azimzadeh I, Afghan M, Momenan AA, Bagheripour F, Azizi F. Pediatric reference values for serum creatinine and estimated glomerular filtration rate in Iranians: Tehran Lipid and Glucose Study. Arch Iran Med 2015;18:753-9.

11. Chang FY, Hwang B, Chen SJ, Lee PC, Meng CC, Lu JH. Characteristics of Kawasaki disease in infants younger than six months of age. Pediatr Infect Dis J 2006;25:241-4.

12. Huang HP, Lai YC, Tsai IJ, Chen SY, Cheng CH, Tsau YK. Nephromegaly in children with Kawasaki disease: new supporting evidence for diagnosis and its possible mechanism. Pediatr Res 2008;63:207-10.

13. Tsau YK, Jou ST, Ni YH. Nephromegaly relates to hepatocyte growth factor dysregulation in biliary atresia. Pediatr Nephrol 2002;17:554-9.

14. Uehara R, Yashiro M, Hayasaka S, et al. Serum alanine aminotransferase concentrations in patients with Kawasaki disease. Pediatr Infect Dis J 2003;22:839-42.

15. Eladawy M, Dominguez SR, Anderson MS, Glodé MP. Abnormal liver panel in acute kawasaki disease. Pediatr Infect Dis J 2011;30:141-4.

16. Newburger JW, Takahashi M, Gerber MA, et al.; Committee on Rheumatic Fever, Endocarditis, and Kawasaki Disease, Council on Cardiovascular Disease in the Young, American Heart Association. Diagnosis, treatment, and long-term management of Kawasaki disease: a statement for health professionals from the Committee on Rheumatic Fever, Endocarditis, and Kawasaki Disease, Council on Cardiovascular Disease in the Young, American Heart Association. Pediatrics 2004;114:1708-33.

17. Kidney Disease: Improving Global Outcomes (KDIGO) Acute Kidney Injury Work Group. KDIGO Clinical Practice Guideline for Acute Kidney Injury. Kidney Int 2012;2:1-138.

18. Lin MT, Wu MH. Coronary diameters in Taiwanese children younger than 6 years old: Z-score regression equations derived from body surface area. Acta Cardiol Sin 2014;30:266-73.

19. Chu LW, Lu MY, Tsau YK. Sonographic measurements of renal size in normal children and children with compensatory renal hypertrophy. Acta Paediatr Taiwan 1999;40:18-21. 